\title{
How community size affects survival chances in cyclic competition games that microorganisms play
}

\author{
Ana Paula O. Müller ${ }^{1}$ and Jason A. C. Gallas ${ }^{1,2,3}$ \\ ${ }^{1}$ Instituto de Física, Universidade Federal do Rio Grande do Sul, 91501-970 Porto Alegre, Brazil \\ ${ }^{2}$ Departamento de Física, Universidade Federal da Paraíba, 58051-970 João Pessoa, Brazil \\ ${ }^{3}$ Instituto de Investigaciones Físicas, Universidad Mayor de San Andres, Cota Cota, La Paz, Bolivia
}

(Received 23 July 2010; published 4 November 2010)

\begin{abstract}
Cyclic competition is a mechanism underlying biodiversity in nature and the competition between large numbers of interacting individuals under multifaceted environmental conditions. It is commonly modeled with the popular children's rock-paper-scissors game. Here we probe cyclic competition systematically in a community of three strains of bacteria Escherichia coli. Recent experiments and simulations indicated the resistant strain of E. coli to win the competition. Other data, however, predicted the sensitive strain to be the final winner. We find a generic feature of cyclic competition that solves this puzzle: community size plays a decisive role in selecting the surviving competitor. Size-dependent effects arise from an easily detectable "period of quasiextinction" and may be tested in experiments. We briefly indicate how.
\end{abstract}

DOI: 10.1103/PhysRevE.82.052901

PACS number(s): 87.23.Cc, 02.50.Ey, 05.40.-a, 64.60.an

An outstanding open question in ecology is to understand the mechanisms responsible for maintaining the overwhelming biodiversity observed in nature [1-5]. A particularly well-documented and appealing mechanism believed capable of maintaining biodiversity is cyclic competition among individuals [6]. A popular example of cyclic competition is the relation among the three strains of $E$. coli [7], highlighted by the possibility of experimentally controlling variables involved in the process.

Basically, cyclic competition in E. coli proceeds as follows. A killer $K$ strain produces and is immune to a toxin (colicin), while colicin-sensitive $S$ bacteria die when in contact with the toxin. Some sensitive bacteria are observed to experience mutations that allow them to become resistant to toxins, creating a resistant strain $R$. Killers $K$ reproduce at a lower pace, due to the metabolic overhead of production and resistance to the toxin, $R$ has an intermediate metabolic cost (it only needs to protect from toxins), while $S$ has the lowest metabolic cost because it neither produces nor needs to defend from toxins. Thus, $S$ wins $R$ because it reproduces faster, $R$ wins $K$ also because it reproduces faster, while $K$ beats $S$ because it produces the toxin that kills $S$. This cycle characterizes rock-paper-scissors-type game competition.

The above relationship makes the $E$. coli strains excellent prototypes to probe mechanisms responsible for maintaining biodiversity under cyclic competition. One of the experimentally tested aspects influencing asymptotic survival is the spatial scale of the interactions, implemented by placing the $K-S-R$ community in different environments as done by Kerr et al. [8]. Following these authors, we refer to phenomena observed in static plate or mixed medium as short- and longrange interactions. Accordingly, short-range interactions were studied by growing bacteria in static plates. For longrange interactions, bacteria were grown either in a shaken flask containing liquid medium or on a mixed plate where every $24 \mathrm{~h}$ a sterile velvet is pressed and rotated against the plate. Under short-range interaction Kerr et al. [8] observed coexistence of all three strains while under long-range interactions only the resistant strain $R$ survived.
The model used to reproduce the cyclic competition of $E$. coli $[8,9]$ consists of a square cellular automaton containing $N \times N$ sites and periodic boundary conditions as usual. Individual sites may assume one of four possible states: a state for each of the $S, R$, and $K$ strains and an empty state. Local interactions are based on the state of the eight nearest neighbors, while long-range interactions are based on eight sites randomly selected from the lattice.

The temporal evolution is done by randomly selecting sites from the lattice and updating them. A sequence of $N^{2}$ random updates is taken as defining the unit of time because, on the average, this is equivalent to updating all sites of the lattice. When a randomly selected site is empty, it is filled by one of the three strains with a probability proportional to the number of individuals of each strain in the neighborhood. Thus, finding zero sites of a given strain ensures that empty sites will not be filled with that strain, thereby guaranteeing that extinct strains remain extinct. The probability of death for occupied sites depends on the strain living in it: $R$ and $K$ die with constant probabilities, $\Delta_{R}$ and $\Delta_{K}$, respectively. However, if a site is occupied by an $S$ strain, the probability of it to die leaving the site empty is proportional to $f_{K}$, the quantity of killer strain $K$ in the neighborhood, according to the equation

$$
\Delta_{S}=\Delta_{S, 0}+\tau f_{K},
$$

where $\tau$ stands for the "toxicity" produced by $K$. To ensure that the model indeed represents cyclic competition the parameters must obey the following relation [8]:

$$
\Delta_{S, 0}<\Delta_{R}<\Delta_{K}<\frac{\Delta_{S, 0}+\tau}{1+\tau} .
$$

As done in previous works $[8,10]$, the effect of different metabolic costs on the reproduction rate is modeled by varying the death rates. We use the same parameter values used by these authors, namely, $\Delta_{S, 0}=1 / 4, \Delta_{R}=10 / 32, \Delta_{K}=1 / 3$, and $\tau=3 / 4$. Initially, the lattice is filled randomly with the four possible states with equal probability. 
Using the above model, Kerr et al. [8] observed good agreement between computer simulations and their biological experiments. Local interactions produced coexistence of the three strains as desired, while just the $R$ strain survived for long-range interactions. In an interesting subsequent work, Károlyi et al. [10] addressed long-range interactions using a mean-field approximation. Surprisingly, mean-field theory predicts the winner to be $S$, the sensitive strain, at variance both with experiments and simulations. To explain such discrepancy, Károlyi et al. argued that the experimental mixing could have been either not homogeneous or not strong enough to reproduce the mean-field expectations. To probe a possible lack of homogeneity they considered chaotic mixtures with a parameter controlling the mixing frequency.

By systematic simulations, we discovered that cyclic competition has a characteristic quasiextinction period, namely, a latency period observed in the mean-field solutions, during which the winning strain $S$ remains smaller than the other strains. This quasiextinction implies that the critical factor controlling the final strain surviving is actually the size of the community. With hindsight, one then realizes a critical difference between the previous simulations. While Kerr et al. simulated a relatively small community of $250 \times 250$ sites, Károlyi et al. considered larger communities, usually with more than $800 \times 800$ sites. So, instead of a discrepancy, both works report behaviors which are typical of the community size that they investigate. Interesting anomalies dependent on the lattice size were recently observed in other models of cyclic competition [11,12], and for particular cyclic relations it is possibly to find a critical population size above which coexistence is likely [13]. We now proceed to present the key point characterizing the quasiextinction period: how big is the impact of the community size in selecting the final surviving strain?

Figure 1(a) displays what happens with the survival probability of strains $R$ and $S$ as a function of the community size when using a global neighborhood. As it is clear from the figure, the surviving strain is quite strongly affected by the lattice size. While for small lattices the prevailing behavior is the survival of the $R$, for larger lattice sizes it is $S$ that survives. Furthermore, for lattices with about $375 \times 375$ sites we find a clear crossover of the strain surviving with highest probability. Thus, the crossover lattice size $N_{c}=375$ is the boundary between two characteristic phases of the model: the resistant and the sensitive phases. By comparing Fig. 1(a) and the experimental results [8], where only $R$ survives, we see that simulations agree with experiments only for small lattice sizes.

We have also investigated size effects when the community is subjected to a chaotic mixing with frequency $\nu$, following the same procedure of Károlyi et al. [10] [see their Eq. (3)]. Figure 1(b) shows the impact of the mixing frequency $\nu$ on the crossover lattice size $N_{c}$, where the crossover occurs. Compared with the result for the global neighborhood [Fig. 1(a)], Fig. 1(b) shows clearly that crossovers occur now for smaller lattice sizes, being, however, present even when mixing is not sufficiently homogeneous to be represented by the mean-field model. In a nutshell, we find crossover to happen for both global neighborhood and for
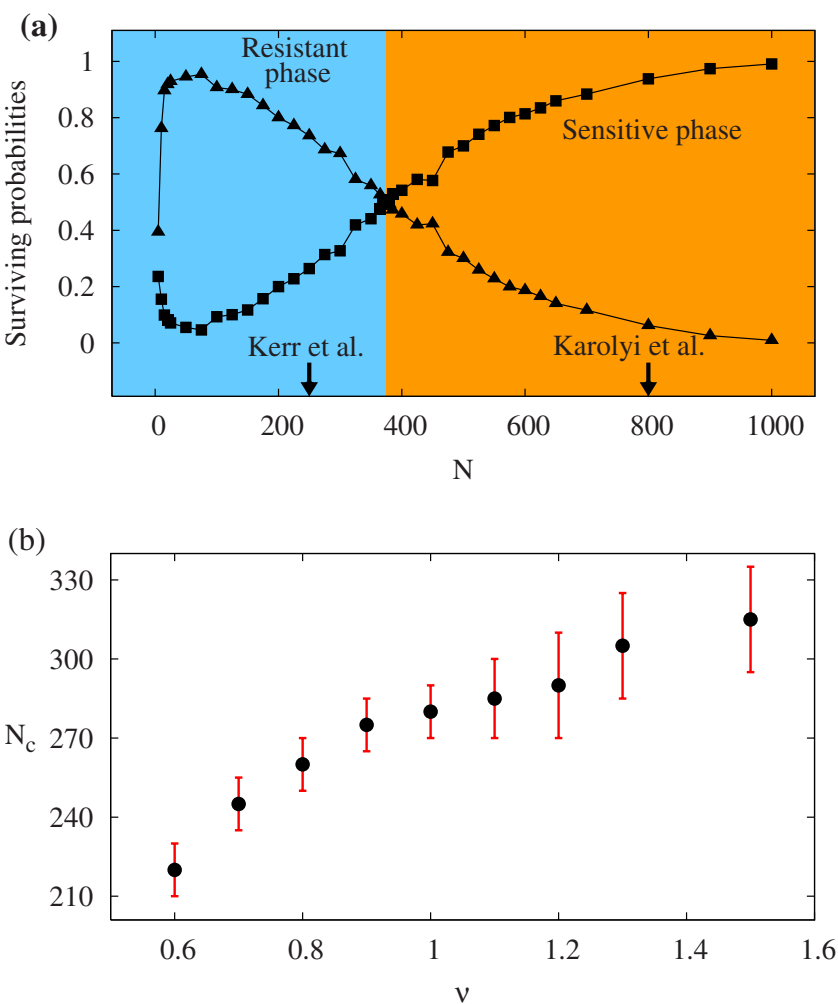

FIG. 1. (Color online) Dependence of the surviving strain as a function of the lattice size $N$. (a) Survival probability of $R$ (triangles) and $S$ (squares) for global neighborhood. Probabilities are averages of 1000 independent simulations on lattices with $N \times N$ sites, starting from random initial conditions. For each set of initial conditions, the fraction of survival was determined by following the time evolution until just one strain remains in the lattice. The $K$ strain survives just for very small lattices (smaller than $25 \times 25$ sites), being omitted from the graph. (b) Evolution of the crossover lattice size, $N_{c}$, as a function of $\nu$, the mixing frequency introduced by Károlyi et al. [10]. To determine $N_{c}$, for each value of $\nu$ we computed a graph like the one in Fig. 1(a) and located the crossing. Each value of $N_{c}$ represents an average over 200 independent sets of initial conditions. Error bars reflect the fact that crossovers occur within a finite interval, not in well-defined points.

chaotic mixing. Therefore, the presence of crossovers must be directly connected to the dynamics of the model and not to the mixing procedure used. In other words, chaotic mixing alone does not explain the experimentally observed survival of $R$.

Figure 2 presents a comparison of typical results about the temporal evolution of the fraction of each bacterial strain as predicted by the mean-field model (thin lines) and as predicted by simulations on a $200 \times 200$ network with global neighborhood (thick lines). The typical behavior for this lattice size is the survival of $R$ strain, but in order to compare with the mean-field results, we used an initial condition for which $S$ survived. It is important to realize that, conceptually, a model with well mixed individuals and finite interaction length is not identical with the mean-field model (described by a set of ordinary differential equations) based on the mean-field interactions [14]. However, despite the small lattice size studied in Fig. 2, for $t<25$ both models agree, pre- 


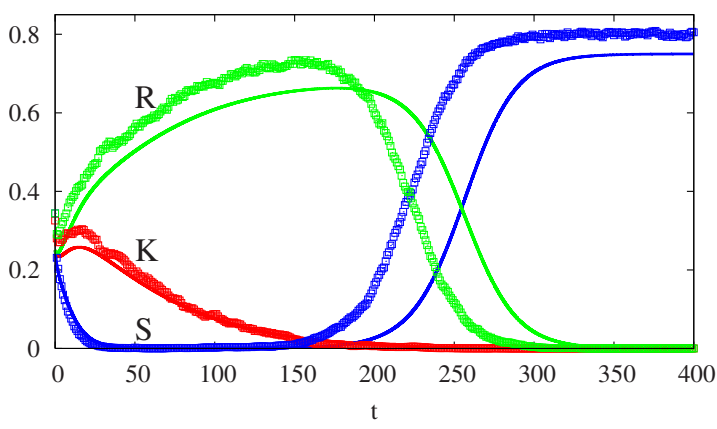

FIG. 2. (Color online) Identification of the quasiextinction period. Temporal evolution of each strain of E. coli according to the mean-field model (thin lines) and according to simulations on a lattice with $200 \times 200$ sites (thick lines). In both cases, during the interval $25 \leq t \leq 150$ the $S$ strain comes very close to zero, characterizing a period of quasiextinction.

dicting a quasiextinction of $S$, when $S$ gets closer to the absorbing state of extinction. Around $t=150$, the $S$ strain starts to grow in both models and, in fact, ends up winning the competition. In small lattices, the quasiextinction of $S$ may, in fact, become a total extinction, allowing $R$ to win the competition. Considering that the fraction of sites occupied by $S$ can assume values as low as $10^{-4}$, in small lattices this fraction can represent zero sites and having reached zero, recovering is no longer possible. This is a strong indication that the quasiextinction of $S$ accounts for the presence of crossovers. For small lattices, $S$ is the strain more likely to get extinct thereby allowing $R$ to win the competition.

For some competition models, extinction may be associ-
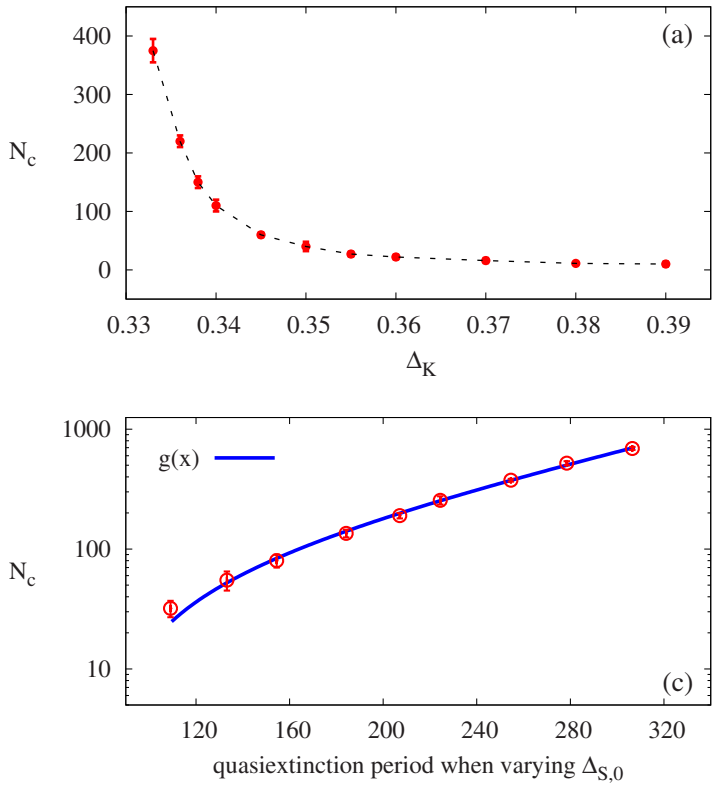

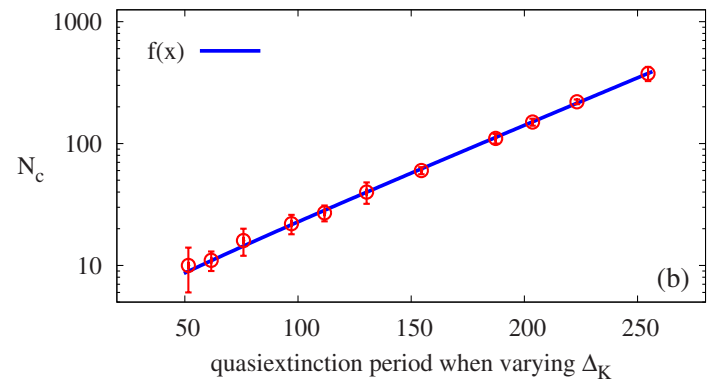

ated to stochastic fluctuations and survival probabilities for each competitor as a function of system size can be determined analytically from stochastic fluctuations [12,13]. The key feature allowing the derivation of analytical results in such models is the existence of a constant of motion depending on parameters and densities of each competitor. In our case, however, no conserved quantity exists due to the presence of empty sites in the lattice, and no analogous derivations are possible.

A simple experiment may be performed to see that the key for the crossovers is the existence of quasiextinction period. In this experiment, we first vary $\Delta_{K}$, which controls the death rate of $K$, and record the crossover lattice size $N_{c}$. As shown in Fig. 3(a), smaller $\Delta_{K}$ corresponds to larger $N_{c}$. Thus, smaller $\Delta_{K}$ shows larger quasiextinction period. Figure 3(b) illustrates for the same $\Delta_{K}$ plotted in Fig. 3(a) how the quasiextinction period affects $N_{c}$. This figure shows clearly that $N_{c}$ grows with the quasiextinction period. Analogously, Figs. 3(c) and 3(d) show a similar growth of $N_{c}$ as a function of quasiextinction period obtained when varying $\Delta_{S, 0}$ and $\Delta_{R}$, respectively. These figures show unambiguously that the growth of $N_{c}$ with the quasiextinction period is a robust feature of the model and that the exponential growth of the three curves is surprisingly governed by very similar exponents. Thus, such behaviors show that the length of the quasiextinction period has a direct connection with crossovers. The slightly distinct behaviors seen in Figs. 3(b)-3(d) might be due to the fact that we are not quantifying the smallness of $S$ during the quasiextinction period. However, this effect is of secondary importance here.

The consistent dynamics described above reveals a ge-

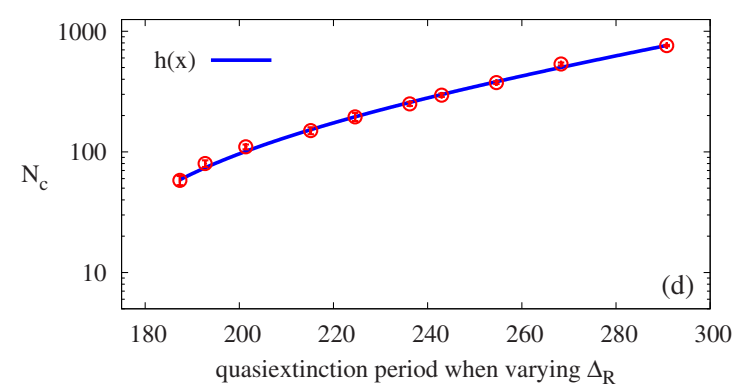

FIG. 3. (Color online) Effect of quasiextinction period of $S$ on the crossover lattice size $N_{c}$. (a) $N_{c}$ as a function of $\Delta_{K}$, the death rate of killer $K$. Variation of $\Delta_{K}$ affects strongly the crossover position in Fig. 1(a) since to decrease $\Delta_{K}$ implies an increase in the resistant phase $R$. (b) $N_{c}$ as a function of the quasiextinction period, calculated for the same eleven values of $\Delta_{K}$ shown in Fig. 3(a). The behavior is well approximated by an exponential: $f(x) \simeq-1.1+4.0 \exp (0.011 x)$. (c) $N_{c}$ computed similarly as in Fig. 3(b) but for nine values of $\Delta_{S, 0}$. The behavior is well approximated by an exponential: $g(x) \simeq-72.9+31.0 \exp (0.017 x)$. (d) $N_{c}$ computed as before for ten values of $\Delta_{R}$. The behavior is well approximated by an exponential: $h(x) \simeq-111.8+8.8 \exp (0.015 x)$. The metric similarity of the three exponentials suggests that the growth of $N_{c}$ is a general feature that does not depend of the parameter changed. 
neric feature of cyclic competition: community size plays a decisive role in selecting the surviving competitor, a feature that solves the puzzling results in the literature. Following the quasiextinction of $S$ in Fig. 2 it is possible to recognize that the recovery of $S$ is caused by the cyclic nature of the competition. As illustrated in Fig. 2, for very low $S$ values $R$ start to dominate the competition since it is only displaced by $S$, the strain that is virtually absent. However, an increase of $R$ means a strong reduction of $K$ since $R$ wins $K$. But with just a few $K$ strains left in the lattice there is no much toxin to prevent the growth of $S$. A decrease in $K$ allows the quasiextinct $S$ to recover and to win the competition. Thus, recovery of $S$ is only possible because of the cyclic nature of the competition, where reduction of one strain strongly affects all the dynamics.

The spatial scale of the interactions is already known as an important factor for the outcome of cyclic competitions $[8,9,15-17]$. To it, we now add a key feature, the lattice size, which manifests through the quasiextinction period. Generic models of cyclic competition support a number of counterintuitive effects such as, for example, unexpected situations where the weakest strain ends winning the competition [18] or the impact of mobility in the loss of diversity [17]. Basically, such counterintuitive effects are generated because competitors are strongly connected, and an important loss or gain in one point of the competition chain affects all individuals. We believe the quasiextinction underlying crossovers to be another important characteristic effect of cyclic relations among competitors that is worth investigating in other models.
To conclude, we mention that lattice-size effects on the coexistence and survival in E. coli may be tested in the laboratory, in experiments with mixed plate. One may equally well vary the size of the dish to uncover lattice-size dependences in the surviving strain. One interesting development that is open to experimentation is based on the fact that, so far, the standard parameters chosen in simulations were not directly related to any biological quantity, except for the fact that they obey the cycling relation described by Eq. (2). As shown in Fig. 3(a), reducing the parameter $\Delta_{K}$ enhances the lattice-size effect in defining the final survivor of the cyclic game. This fact suggests that size effects may be much stronger in real experiments than those observed here. Knowing that the number of individuals is huge in experimental situations and that simulations and the mean-field limit predict survival of $S$, it is somehow surprising that Kerr et al. found the $R$ strategy to win in the experiment. There are several possible explanations for this fact, ranging from shortcomings in the modeling, e.g., using sites to describe individuals instead of colonies, to unaccounted perturbations during experimental measurements. This puzzle certainly requires further investigation.

J.A.C.G. thanks Professor G. M. Ramírez-Ávila for his interest and hospitality in La Paz. The authors are supported by CNPq, Brazil, and US-AFOSR Grant No. FA9550-07-10102. They also thank CESUP-UFRGS for granting computer time.
[1] A. R. Ives and S. R. Carpenter, Science 317, 58 (2007).

[2] M. A. Nowak and K. Sigmund, Science 303, 793 (2004).

[3] P. Chesson, Annu. Rev. Ecol. Syst. 31, 343 (2000).

[4] J. M. Levine and J. HilleRisLambers, Nature (London) 461, 254 (2009).

[5] J. M. Smith, Nature (London) 380, 198 (1996).

[6] H. Shi, W. X. Wang, R. Yang, and Y. C. Lai, Phys. Rev. E 81, 030901(R) (2010); D. L. Roelke and P. M. Eldridge, Ecol. Modell. 221, 1017 (2010); D. D. Cameron, A. White, and J. Antonovics, J. Ecol. 97, 1311 (2009); T. L. Czárán, R. F. Hoekstra, and L. Pagie, Proc. Natl. Acad. Sci. U.S.A. 99, 786 (2002); B. Sinervo and C. M. Lively, Nature (London) 380, 240 (1996); J. B. C. Jackson and L. Buss, Proc. Natl. Acad. Sci. U.S.A. 72, 5160 (1975); R. M. May and W. J. Leonard, SIAM J. Appl. Math. 29, 243 (1975).

[7] T. Reichenbach and E. Frey, Physik Journal 8, 27 (2009); F. Prado and B. Kerr, Evolution (Lawrence, Kans.) 62, 538 (2008); B. C. Kirkup and M. A. Riley, Nature (London) 428, 412 (2004); M. A. Riley and D. M. Gordon, Trends Microbiol. 7, 129 (1999); R. James, C. Kleanthous, and G. R. Moore, Microbiology 142, 1569 (1996); L. Chao and B. R. Levin, Proc. Natl. Acad. Sci. U.S.A. 78, 6324 (1981).
[8] B. Kerr, M. A. Riley, M. W. Feldman, and B. J. M. Bohannan, Nature (London) 418, 171 (2002).

[9] R. Durrett and S. Levin, J. Theor. Biol. 185, 165 (1997).

[10] G. Károlyi, Z. Neufeld, and I. Scheuring, J. Theor. Biol. 236, 12 (2005)

[11] J. Cremer, T. Reichenbach, and E. Frey, Eur. Phys. J. B 63, 373 (2008).

[12] M. Berr, T. Reichenbach, M. Schottenloher, and E. Frey, Phys. Rev. Lett. 102, 048102 (2009).

[13] J. C. Claussen and A. Traulsen, Phys. Rev. Lett. 100, 058104 (2008).

[14] S. Számadó, F. Szalai, and I. Scheuring, J. Theor. Biol. 253, 221 (2008); P. M. Altrock and A. Traulsen, Phys. Rev. E 80, 011909 (2009); A. Traulsen, J. C. Claussen, and C. Hauert, ibid. 74, 011901 (2006); Phys. Rev. Lett. 95, 238701 (2005).

[15] R. A. Laird and B. S. Schamp, Ecology 89, 237 (2008).

[16] M. Peltomäki and M. Alava, Phys. Rev. E 78, 031906 (2008).

[17] T. Reichenbach, M. Mobilia, and E. Frey, Nature (London) 448, 1046 (2007).

[18] M. Frean and E. R. Abraham, Proc. R. Soc. London, Ser. B 268, 1323 (2001). 